\title{
Presence of lipoprotein lipase S447X stop codon affects the magnitude of interleukin 8 release after cardiac surgery with cardiopulmonary bypass
}

\author{
Koray Ak, MD, ${ }^{a}$ Selim İsbir, MD, ${ }^{\text {a }}$ Atike Tekeli, MD, ${ }^{\text {a }}$ Arzu Ergen, PhD, ${ }^{\text {b }}$ Nazan Atalan, MD, ${ }^{\mathrm{c}}$ Selami Dogan, MD, ${ }^{\mathrm{d}}$
} Ali Civelek, MD, and Sinan Arsan, MD ${ }^{\mathrm{a}}$

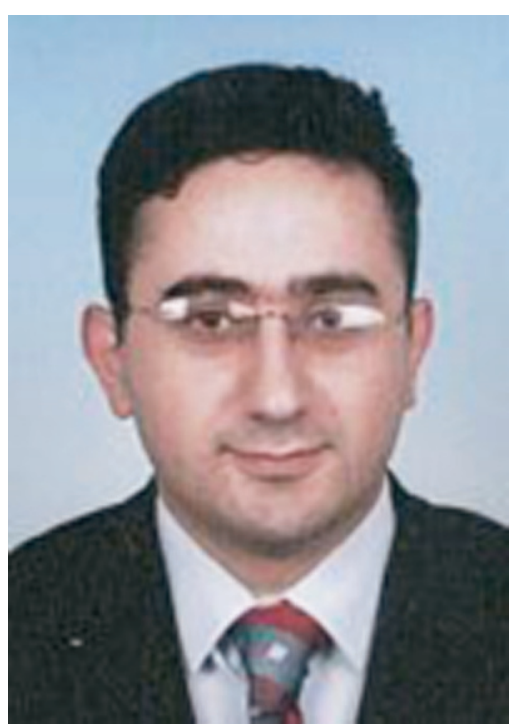

Dr Ak

Objective: Current data suggest that individual genetic predisposition may influence the magnitude of cytokine response and the degree of organ dysfunction after cardiopulmonary bypass. Lipoprotein lipase S447X polymorphism has been shown to be protective against atherosclerosis. The aim of the study was to investigate the effect of lipoprotein lipase S447X polymorphism on cytokine release and early outcome after cardiopulmonary bypass.

Methods: Forty patients who underwent coronary artery bypass grafting with cardiopulmonary bypass were included. Genotyping for lipoprotein lipase S447X polymorphism was performed by polymerase chain reaction. Levels of interleukins 6 and 8 were measured before induction and 6, 24, and 72 hours after operation by enzyme-linked immunosorbent assay. Clinical data were collected prospectively. Daily assessment of organ dysfunction was done according to the cardiac surgery scoring (CASUS) system.

Results: The allele frequency of lipoprotein lipase S447X stop codon was $17.5 \%$. S447X carriers revealed significantly lower interleukin 8 levels at the sixth and 24th postoperative hours than the noncarrier group $(P=.005$ and $P=.041$, respectively). Patients in the S447X carrier group had significantly shorter ventilation times than the noncarrier group $(P=.048)$. Also, the $\mathrm{S} 447 \mathrm{X}$ carrier group revealed significantly lower postoperative 6-hour lactate levels, operative day, and postoperative day 1 organ dysfunction scores than the other group $(P=.001, .005$ and .002 , respectively).

From Marmara University School of Medicine, Department of Cardiovascular Surgery, Istanbul, Turkey, ${ }^{\mathrm{a}}$ Istanbul University School of Medicine, Institute of Experimental Medical Research, Department of Molecular Medicine, Istanbul, Turkey, ${ }^{\mathrm{b}}$ Özel Academic Hospital, Department of Anesthesiology and Reanimation, Istanbul, Turkey, ${ }^{\mathrm{c}}$ and Johann Wolfgang Goethe University, Department of Cardiovascular Surgery, Frankfurt Main, Germany. ${ }^{\mathrm{d}}$

Received for publication Feb 10, 2007; revisions received March 1, 2007; accepted for publication March 12, 2007.

Address for reprints: Koray AK, MD, Marmara University Hospital, 34640 Altunizade Istanbul, Turkey (E-mail: akkoray@ hotmail.com).

J Thorac Cardiovasc Surg 2007;134:477-83

0022-5223/\$32.00

Copyright (C) 2007 by The American Association for Thoracic Surgery

doi:10.1016/j.jtcvs.2007.03.017

Conclusion: Lipoprotein lipase S447X stop codon mutation is associated with lower levels of interleukin 8 after coronary artery bypass grafting. Identification of high-risk patients for cardiopulmonary bypass-related systemic inflammation by detecting lipoprotein lipase S447X stop codon polymorphism may improve early postoperative outcome, especially in patients with limited organ reserves.

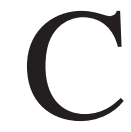

ardiac surgery with cardiopulmonary bypass (CPB) is associated with a systemic inflammatory response syndrome (SIRS) that is known to be one of the main causes of postoperative organ dysfunction. ${ }^{1}$ Several factors such as contact of blood with foreign surfaces, surgical trauma, ischemia reperfusion to the organs, and release of endotoxin have been widely documented to cause SIRS after $\mathrm{CPB} .^{2}$ As a consequence of the inflammatory reaction, a clinical picture ranging from a subclinical increase in the humoral inflammatory mediators to multiple organ dysfunction with lactic acidosis and even death occurs. ${ }^{2,3}$ Current data suggest that the individual genetic predisposition is an important determinant of the degree of postoperative SIRS in cardiac surgery. The magnitude of inflammatory cytokines, particularly interleukin (IL) 6, IL-8, and tumor necrosis factoralpha (TNF- $\alpha$ ) produced during $\mathrm{CPB}$ has been shown to be unfavorably altered in 


Abbreviation and Acronyms
$\begin{aligned} & \text { APACHE }= \text { Acute Physiology and Chronic Health } \\ & \text { Evaluation } \\ & \text { CABG }=\text { coronary artery bypass grafting } \\ & \text { CASUS }=\text { cardiac surgery scoring } \\ & \text { CK }=\text { creatine kinase } \\ & \text { CPB }=\text { cardiopulmonary bypass } \\ & \text { ICU }=\text { intensive care unit } \\ & \text { IL }=\text { interleukin } \\ & \text { MODS }= \text { Multiple Organ Dysfunction Score } \\ & \text { LPL }=\text { lipoprotein lipase } \\ & \text { SIRS }=\text { systemic inflammatory response syndrome } \\ & \text { TNF }=\text { tumor necrosis factor }\end{aligned}$

the presence of IL-6 G174C, apolipoprotein E4, and TNF AA-308 genetic polymorphisms. ${ }^{4-7}$

Lipoprotein lipase (LPL) is an enzyme that catalyses a key step in the removal of triglycerides from the circulation. ${ }^{8}$ Besides its catalytic activity, data have accumulated that LPL has a pivotal role in the inflammatory process of atherosclerosis by modulating the cytokine release. ${ }^{9-11} \mathrm{Sev}$ eral polymorphisms have been described in the gene coding for LPL. Of these, LPL S447X stop codon mutation is regarded as a gain of function mutation and associated with increased peripheral LPL activity and high-density lipoprotein, decreased triglycerides, and the decreased risk of coronary artery disease. ${ }^{12,13}$

We hypothesized that the genotype of the S447X polymorphism in the $L P L$ gene may dictate the IL-6 and IL-8 levels and therefore influence the inflammatory response after coronary artery bypass grafting (CABG). We investigated the relationship between the LPS-S447X polymorphism and the magnitude of postoperative IL-6 and IL-8 response and organ dysfunction in patients undergoing CABG with CPB.

\section{Materials and Methods}

After obtaining approval to conduct the study from the local ethics committee and written informed consent from each patient, we enrolled 40 consecutive patients undergoing elective CABG into the study. Exclusion criteria were unstable angina pectoris, previous CABG, known cancer, known infection, steroid or nonsteroidal anti-inflammatory therapy, an autoimmune condition, preoperative intra-aortic balloon pump insertion, emergency revascularization, long-term dialysis, and hepatic failure. Sixty-six healthy control individuals (aged between 35 and 60 years) were used to investigate genotype distribution and allele frequencies in comparison with patients. According to the results of medical history, physical examination, and laboratory data, they were judged healthy.

Medical history, demographic data, and the postoperative course for each patient were collected prospectively. Preoperative evaluation included routine blood biochemistry, complete blood count, pulmonary function test, transthoracic echocardiography, and coronary angiography. In the postoperative period, patients were followed up and treated according to the same institutional protocol.

Postoperative daily assessment of organ function in the intensive care unit (ICU) was performed by the cardiac surgery scoring (CASUS) system ${ }^{14}$ (Appendix 1). In brief, CASUS contains 10 variables that were graded according to the severity for daily risk stratification in the ICU. Operative day CASUS score was calculated when the patient was admitted to the ICU. Then, subsequent calculations were performed in the morning of each ICU day. Perioperative myocardial infarction was defined by new electrocardiographic changes and an increase of 3.5-fold in creatine kinase (CK) isoenzyme MB level. Need for an inotropic agent was judged by systolic blood pressure lower than $90 \mathrm{~mm} \mathrm{Hg}$ or more than $40 \mathrm{~mm} \mathrm{Hg}$ below usual systolic pressure under appropriate fluid management. Postoperative acute renal dysfunction was defined as a postoperative serum creatinine level greater than 200 $\mu \mathrm{mol} / \mathrm{L}$ or need for dialysis therapy or hemofiltration before hospital discharge.

All operations were performed by the senior surgeon and two senior residents. The surgical team and the anesthesiologist who were responsible for the postoperative care of the patient were blinded to the presence or absence of LPL S447X stop codon.

\section{Anesthesia and Operative Technique}

Midazolam was used for premedication, and the anesthetic agent consisted of a combination of fentanyl, midazolam, and pancuronium. Anesthesia was maintained with midazolam and vecuronium infusion and with inhaled sevoflurane. Median sternotomy was carried out in all patients. Standard aortocaval cannulation was done to establish CPB. CPB was performed with a roller pump system (Jostra AG, Hirrlingen, Germany) and a hollow-fiber membrane oxygenator (Jostra Quadrox, Hirrlingen, Germany). Also, a $40-\mu \mathrm{m}$ arterial blood filter (Jostra AG) was used in all patients. Mild-to-moderate $\left(28^{\circ} \mathrm{C}-32^{\circ} \mathrm{C}\right)$ hypothermia and pulsatile flow of 2.2 to $2.4 \mathrm{~L} / \mathrm{m}^{2}$ were maintained throughout $\mathrm{CPB}$ in all patients. Myocardial protection was provided by antegrade tepid blood cardioplegic solution and topical cooling during aortic crossclamping. Repeated infusions of $300 \mathrm{~mL}$ were given every 20 minutes or earlier if electrical activity occurred. Rewarming to a normal nasopharyngeal temperature was achieved with a heat-exchange oxygenator and warming blanket. Left internal thoracic artery and saphenous vein were preferred as bypass conduits in all patients. The rest of the operation was completed in a standard fashion. Perioperative anticoagulation with heparin was reversed after $\mathrm{CPB}$ with protamine sulfate. Aprotinin and steroids were not used.

\section{Biochemical Measurements}

Leukocyte count of the blood samples was determined by the use of the Coulter system (Coulter HMX-AL system hematology analyzer; Beckman Coulter Corporation, Miami, Fla) before induction, immediately after $\mathrm{CPB}$, and 24 hours after the operation. Lactate levels were analyzed (GEM Premier 3000 blood gas/ electrolyte analyzer model 5700; Instrumentation Laboratory, Lexington, Mass) from radial artery blood samples taken at preoperatively, immediately after CPB, and 6, 12, and 24 hours postoperatively. CK (Cobas Integra 700; Roche Diagnostics, 
TABLE 1. Genetic profile of control subjects and patients

\begin{tabular}{lccc}
\hline & $(+/+)$ & $(-/+)$ & $(-/-)$ \\
\hline Controls $(n=66)$ & $1(1.5)^{*}$ & $8(12.1)$ & $57(86.4)$ \\
Patients $(n=40)$ & 0 & $7(17.5)$ & $33(82.5)$
\end{tabular}

The symbols $(-/-),(-/+)$, and $(+/+)$ stand for homozygote and heterozygote for the absence of the lipoprotein lipase S447X stop codon and homozygote for the presence of the lipoprotein lipase S447X stop codon, respectively. ${ }^{*}$ Numbers in parenthesis indicate percentage.

Basel, Switzerland) and CK-MB levels (Elecsys 2010; Roche Diagnostics) were measured preoperatively and 6 and 24 hours after the operation.

\section{IL-6 and IL-8 Levels}

Levels of IL-6 and IL-8 were measured by enzyme-linked immunosorbent assay (Cat No: KHC0061 and KHC0081; Invitrogen, BioSource Division, Carlsbad, Calif) according to the manufacturer's recommendations. The arterial blood samples were collected from the radial artery catheter into sterile ethylenediaminetetraacetic acid tubes. After centrifugation, the plasma was collected and then stored at $-20^{\circ} \mathrm{C}$ until biochemical analysis. Levels of IL-6 and IL- 8 were measured at 4 different time points: $\mathrm{t} 1$, before induction of anesthesia; t2, 6 hours after operation; t3, 24 hours after operation; and t4, 72 hours after operation.

\section{Isolation of DNA and LPL Genotyping}

Isolation of DNA and LPL genotyping were performed as described. ${ }^{15}$ Whole blood samples for genotyping were drawn preoperatively from both patients and healthy control individuals. LPL gene S447X (refsnp 328) genotype was determined by polymerase chain reaction restriction fragment length polymorphism analysis using primers 5'-CATCCATTTTCTTCCACAGGG-3' (forward) and 5'-TAGCCCAGAATGCTCACCAGACT-3' (reverse) to generate a polymerase chain reaction product of $137 \mathrm{bp}$, which, in the presence of the X447 allele, is digested to 114 and 23 bp by HinfI (New England Biolabs, Ipswich, Mass). Fragments were separated by electrophoresis on a $6 \%$ horizontal polyacrylamide gel by microtiter array diagonal gel electrophoresis. ${ }^{16}$

\section{Statistical Analysis}

Statistical analysis was performed with the SPSS for Windows 10.0 version (SPSS, Inc, Chicago, Ill). The $\chi^{2}$ test was used to analyze relationships between categorical data. Nonparametric Mann-Whitney $U$ test was used to compare IL-6 and IL-8 levels and different outcomes between groups. Nonparametric testing was chosen because the study population was relatively small in size and the data did not follow a normal distribution. Association between two continuous variables was determined by Spearman rank correlation. Continuous variables were given as mean \pm standard error of the mean. A $\chi^{2}$ test was used to compare the observed numbers of each genotype with those expected for a population to establish whether they were in the Hardy-Weinberg equilibrium. Regression analyses were used to adjust for confounding factors. All tests were 2-sided. All measurements between and within the groups were checked for different time points
TABLE 2. Preoperative characteristics of the patients

\begin{tabular}{|c|c|c|c|}
\hline & $\begin{array}{c}\text { S447X } \\
\text { noncarriers } \\
(\mathrm{n}=33)\end{array}$ & $\begin{array}{c}\text { S447X } \\
\text { carriers } \\
(\mathrm{n}=7)\end{array}$ & $P$ value \\
\hline Age & $58.04 \pm 7.82$ & $58 \pm 2.70$ & .986 \\
\hline Sex (male/females) & $28 / 5$ & $5 / 2$ & .396 \\
\hline $\operatorname{BSA}\left(\mathrm{m}^{2}\right)$ & $1.74 \pm 0.15$ & $1.80 \pm 5.46$ & .897 \\
\hline Smoker & $17(55.5) *$ & $2(28.5)$ & .447 \\
\hline DM & $10(30.3)$ & $3(42.8)$ & .519 \\
\hline HT & $17(55.5)$ & $3(42.8)$ & .667 \\
\hline COPD & $9(27.2)$ & $2(28.5)$ & .760 \\
\hline Hyperlipidemia & $14(42.4)$ & $3(42.8)$ & .983 \\
\hline Ejection fraction (\%) & $53.16 \pm 7.36$ & $61.42 \pm 2.82$ & .013 \\
\hline \multicolumn{4}{|l|}{ MI history } \\
\hline Recent (<3 weeks) & $4(12.1)$ & $1(14.2)$ & .875 \\
\hline Remote (>3 weeks) & $8(24.2)$ & $2(28.5)$ & .810 \\
\hline \multicolumn{4}{|l|}{ Preoperative drugs } \\
\hline Beta-blocker & $14(42.4)$ & $3(42.8)$ & .983 \\
\hline ACE inhibitors & $5(15.1)$ & $1(14.2)$ & .954 \\
\hline EuroSCORE & $2.13 \pm 0.3$ & $2.35 \pm 0.4$ & .421 \\
\hline
\end{tabular}

$D M$, Diabetes mellitus; $H T$, hypertension; $C O P D$, chronic obstructive pulmonary disease; $M I$, myocardial infarction; $A C E$, angiotensin-converting enzyme.

by repeated-measures analysis of variance with Bonferroni corrections for multiple testing.

\section{Results}

Genetic characteristics of patients and control subjects were summarized in Table 1 . Of the 40 patients enrolled in the study, 7 (17.5\%) patients revealed heterozygote pattern for the presence of the S447X stop codon $(-/+)$, which was found to be similar to the control population (12.1\%). Only 1 control subject revealed homozygote pattern for the presence of the LPL S447X stop codon $(+/+)$. The allele frequency of the LPL S447X allele did not deviate from Hardy-Weinberg expectations $(P=.560)$.

Comparison of the demographics and the preoperative profile of the S447X carriers $(-/+)$ and the noncarriers $(-/-)$ revealed no difference (Table 2$)$. There was no correlation between the incidence of hyperlipidemia and the S447X genetic variant. All patients had significant triplevessel coronary artery disease and normal renal function preoperatively. Intraoperatively, aortic crossclamp time, $\mathrm{CPB}$ time, and the number of performed distal anastomoses did not differ between the two groups (Table 3 ). The postoperative data demonstrated that ventilation time was significantly longer in the S447X noncarrier group than the other group $(P=.048)$. The ICU stay was slightly longer in S447X noncarriers than the other group but the difference was not statistically significant (33.64 \pm 3.09 hours vs $20.42 \pm 1.08$ hours; $P=.074$ ). Inotropic drug requirement, chest tube drainage, perioperative transfusion, and hospital stay did not differ between the groups. Leukocyte counts 
TABLE 3. Relationship between lipoprotein lipase gene polymorphism and perioperative variables

\begin{tabular}{lccc}
\hline & S447X noncarriers $\mathbf{( n = 3 3 )}$ & S447X carriers $(\mathbf{n}=\mathbf{7})$ & $\boldsymbol{P}$ value \\
\hline ACC time (min) & $36.39 \pm 1.01$ & $37 \pm 2.70$ & .681 \\
CPB time (min) & $69.60 \pm 1.59$ & $74.28 \pm 4$ & .318 \\
No. of grafts & $3.42 \pm 0.12$ & $3.57 \pm 0.36$ & .600 \\
Perioperative MI & $1(3)$ & 0 & .641 \\
Ventilation time (h) & $6.78 \pm 0.56$ & $4.71 \pm 0.28$ & $.048 *$ \\
Need for inotropic agents & $5(15.1)$ & $1(14.2)$ & .954 \\
Blood loss 24 hours (mL) & $504.54 \pm 36.95$ & $521.42 \pm 77.04$ & .747 \\
Perioperative transfusion & $5(15.1)$ & $1(14.2)$ & .954 \\
Packaged RBC & $2(6)$ & 0 & .504 \\
FFP & $1(3)$ & 0 & .641 \\
TS & $33.64 \pm 3.09$ & $20.42 \pm 1.08$ & .074 \\
ICU stay (h) & $2(6)$ & 0 & .504 \\
Postoperative AF & $5.81 \pm 0.97$ & $5.42 \pm 0.36$ & .464 \\
Hospital stay (d) & $2(6)$ & 0 & .504 \\
Postoperative ARD & $1(3) / 0$ & 0 & .641 \\
Hemofiltration/hemodialysis & & $4.06 \pm 0.38$ & .005 \\
CASUS scoring & $6.85 \pm 3.23$ & $4.21 \pm 0.60$ & .002 \\
Operative day & $5 \pm 2.38$ & & \\
Postoperative day 1 & & & \\
\hline
\end{tabular}

$A C C$, Aortic crossclamp; $C P B$, cardiopulmonary bypass; $M I$, myocardial infarction; $R B C$, red blood cells; FFP, fresh frozen plasma; TS, thrombocyte suspension; ICU, intensive care unit; $A F$, atrial fibrillation; $A R D$, acute renal dysfunction; CASUS, cardiac surgery scoring. *Boldface $P$ values indicate significance.

before induction, immediately after $\mathrm{CPB}$, and 24 hours after operation were similar between the two groups $(P=.663$, .722 , and .946 , respectively).

Postoperative daily assessment of organ dysfunction in the ICU revealed that S447X noncarriers showed significantly higher postoperative day 0 (operative day) and day 1 CASUS scores than the carriers $(6.85 \pm 3.23$ vs $4.06 \pm$ $0.38 ; P=.005$; and $5 \pm 2.38$ vs $4.21 \pm 0.60 ; P=.002$, respectively). Owing to the prolonged ICU stay, postoperative day 2 CASUS scores were calculated for only 8 patients in the S447X noncarrier group $(5.75 \pm 0.81)$ and none of the patients in the S447X carrier group. Two patients in the S447X noncarrier group had acute postoperative renal dysfunction and 1 of them required venovenous hemofiltration or dialysis. There was no perioperative mortality in either group.

The S447X noncarrier group revealed significantly higher levels of lactate at the postoperative sixth hour than the other group $(4.76 \pm 0.23$ vs $2.26 \pm 0.26 \mathrm{mmol} / \mathrm{L} ; P=$ $.001)$. Preoperative and postoperative 12- and 24-hour plasma lactate levels were similar between the two groups $(P=.744, .066$, and .216, respectively) (Figure 1).

Preoperative levels of the IL-6 and IL-8 were detectable in almost all patients. The peak IL-6 concentration was detected at the 24th postoperative hour in both groups, and there were no differences in levels of IL-6 at 4 time points (t1 to t4) between the S447X noncarrier group and the S447X carrier group (t1, $6.48 \pm 2.62 \mathrm{pg} / \mathrm{mL}$ vs $8.42 \pm 1.39$ $\mathrm{pg} / \mathrm{mL}[P=.192] ; \mathrm{t} 2,37.55 \pm 20.69 \mathrm{pg} / \mathrm{mL}$ vs $37.04 \pm$
$8.74 \mathrm{pg} / \mathrm{mL}[P=.776] ; \mathrm{t} 3,104.93 \pm 75.39 \mathrm{pg} / \mathrm{mL}$ vs $140.08 \pm 54.82 \mathrm{pg} / \mathrm{mL}[P=.772] ;$ and $\mathrm{t} 4,60.28 \pm 29.7$ $\mathrm{pg} / \mathrm{mL}$ vs $93.68 \pm 44.14 \mathrm{pg} / \mathrm{mL}[P=.873]$ ) (Figure 2, $A$ ). The peak IL-8 concentration was detected at the sixth postoperative hour in both groups (Figure 2, B). There was a significant effect of genotype on IL-8 levels. In the S447X

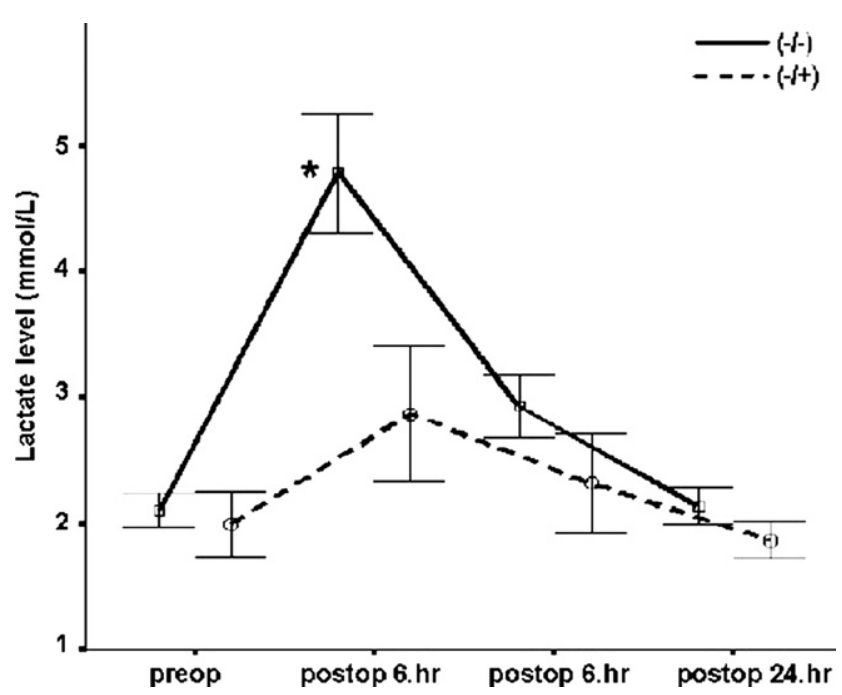

Figure 1. Comparison of lactate levels. The symbols $(-/-)$ and $(-/+)$ stand for homozygote and heterozygote for the absence of the LPL S447X stop codon, respectively. ${ }^{*} P<.05$. Error bars represent the standard error of mean. 

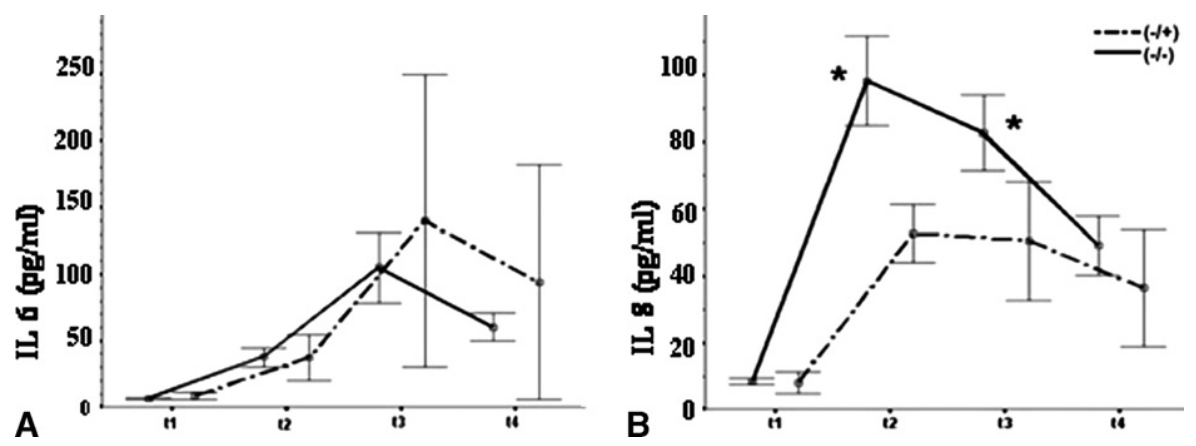

Figure 2. Mean IL-6 (A) and mean IL-8 levels (B) at 4 different time points. The symbols $(-/-)$ and $(-/+)$ stand for homozygote and heterozygote for the absence of the LPL S447X stop codon, respectively. $t 1$, Before the induction of anesthesia; $t 2,6$ hours after operation; $t 3,24$ hours after operation; $t 4,72$ hours after operation. ${ }^{*} \boldsymbol{P}<.05$. Error bars represent the standard error of mean. IL, Interleukin. carrier group, patients had significantly higher IL-8 levels at both 6 hours and 24 hours after operation than S447X noncarriers $(98.33 \pm 38.41 \mathrm{pg} / \mathrm{mL}$ vs $52.85 \pm 4.38 \mathrm{pg} / \mathrm{mL}$; $P=.005$; and $82.82 \pm 5.68 \mathrm{pg} / \mathrm{mL}$ vs $50.57 \pm 8.92 \mathrm{pg} / \mathrm{mL}$; $P=.041$; respectively). The difference became nonsignificant at 72 hours after operation between the groups $(49.18 \pm 24.95 \mathrm{pg} / \mathrm{mL}$ vs $36.42 \pm 8.69 \mathrm{pg} / \mathrm{mL} ; P=.421)$.

\section{Discussion}

Our results showed a highly significant positive correlation between postoperative 6- and 24-hour IL-8 levels and the absence of the S447X stop codon. Levels of IL-6 did not reveal a difference according to the genotype. Additionally, patients carrying S447X genotype revealed lower postoperative organ dysfunction scores and early postoperative lactate levels and had shorter ventilation time than did the noncarriers. ICU stay was also slightly shorter in S447X carriers.

The use of CPB is still a prerequisite for many cardiac surgical procedures. Cardiac surgery with $\mathrm{CPB}$ provokes an acute phase reaction, SIRS, the pathophysiology of which is quite complex. ${ }^{1-3}$ Even though the SIRS has been shown to be one of the important determinants of postoperative organ dysfunction and mortality, the modulators of the tissue damage are not yet fully clarified. Moreover, it is still difficult to estimate the high-risk patient population for systemic inflammation and consequent organ dysfunction after cardiac operations. Recently, it has been demonstrated that the magnitude of the inflammatory cytokine response and clinical consequences of CPB-related SIRS are genetically controlled and vary from patient to patient. ${ }^{4-6}$ In a study by Guadino and colleagues, ${ }^{4}$ patients with IL-6-174 G/C genotype revealed higher post-CPB IL-6 levels, longer stays in the hospital and in the ICU, and higher degrees of renal and pulmonary complications after CABG. Bittar and colleagues ${ }^{6}$ demonstrated that patients with AA-308 TNF- $\alpha$ genotype showed higher plasma levels of TNF- $\alpha$ and less favorable early outcome than those with AG and GG genotypes after CABG with CPB.

Several polymorphisms at the LPL locus were described, and most of them like the Hind III $\left(\mathrm{H}^{+}\right.$allele $)$, the PvuII $\left(\mathrm{P}^{+}\right.$ allele), the S291 and the Asp9Asn genetic variants have been shown to be unfavorably associated with variations in LPL activity, serum lipid concentrations, and the risk and the progression of coronary artery disease. ${ }^{8,17}$ LPL S447X stop codon mutation is caused by cytosine to guanine transversion at nucleotide 1595 in exon 9 that results in the premature truncation of the LPL enzyme by deleting two amino acids. ${ }^{8,12}$ The carrier frequency of this polymorphism was reported to be $10 \%$ to $25 \%$ in the general population, which was consistent with the frequency in our patient population $(17.5 \%) .^{8}$ The $\mathrm{S} 447 \mathrm{X}$ polymorphism has been shown to be associated with higher plasma LPL catalytic activity, reduced triglycerides, increased high-density lipoprotein cytosine and LPL catalytic activity, and a significantly decreased risk of coronary artery disease. ${ }^{12,13} \mathrm{We}$ could not detect a relationship between the S447X stop codon mutation and hyperlipidemia in this study.

In our patient cohort, patients with LPL S447X stop codon revealed an exaggerated IL-8 response to $\mathrm{CPB}$ whereas the IL-6 response was not altered according to the genotype. Increased levels of IL- 6 and TNF- $\alpha$ have been associated with cardiac dysfunction after $\mathrm{CPB} .^{2}$ However, it is shown that IL-8 is a potent chemoattractant for neutrophils and, therefore, a leading cause for pulmonary leukocyte sequestration and associated lung injury after $\mathrm{CPB}{ }^{3}$ Kotani and colleagues ${ }^{18}$ revealed that IL-8-mediated neutrophil activation is the major cause of CPB-related pulmonary dysfunction, and the number of neutrophils in bronchoalveolar lavage after CBP is significantly correlated with the increases in IL-8 concentrations. Similarly, Grünenfelder and colleagues ${ }^{7}$ reported a positive association between higher postoperative releases of IL- 8 and TNF- $\alpha$ and prolonged intubation in patients having TNF- $\beta$ A329G and apolipoprotein E4 polymorphisms. In accordance with results from the literature, patients with a more pronounced IL-8 response to $\mathrm{CPB}$ had longer ventilation times and higher daily organ dysfunction scores in the present study.

Higher CASUS scores in LPL S447X noncarriers seem to be related to the increased lactate levels, higher rate of pulmonary dysfunction, and slightly prolonged ICU stay. It has been shown that CASUS has more clinical validity in 
critically ill patients in the ICU after cardiac surgery. ${ }^{14}$ This explains why we could not demonstrate a relationship between scoring and clinical outcome. There were no differences in the blood leukocyte counts between the groups in our study. We think LPL S447X genetic variant exerts a resistance against the SIRS and some of its consequences, such as pulmonary dysfunction, by decreasing the magnitude of postoperative IL-8 production after CABG.

Lactate is a widely known marker for the occurrence of anaerobic metabolism resulting from tissue hypoxia or ischemia. Excessive cytokine production during SIRS causes an increase in plasma lactate levels. ${ }^{3}$ Additionally, postoperative lactate level is a good predictor of low cardiac output and failure of intra-aortic balloon pumping in cardiac surgery. ${ }^{19,20}$ It has been demonstrated that IL-8 is one of the most powerful predictors of lactatemia, severe hypoxemia, the Acute Physiology and Chronic Health Evaluation (APACHE) II score, and mortality rate in septic shock. ${ }^{21}$ Rao and colleagues ${ }^{19}$ claimed that increased myocardial lactate release during reperfusion reflects a delayed recovery of aerobic myocardial metabolism. In our study, S447X noncarriers revealed more intense IL- 8 and lactate release at the early postoperative period. However, we could not detect any difference between the two groups in terms of perioperative CK-MB levels and need for inotropic agents. The detrimental effects of increased postoperative lactate and IL-8 levels on the clinical outcome, particularly on myocardial metabolism and function, were not obvious, and this could be related to the well-preserved myocardial function and energy stores preoperatively in this patient cohort. The effect of the genotype-related organ dysfunction would be expected to be more pronounced in patients with more severe comorbidities.

On the basis of current data about the pathophysiology of atherosclerosis, out of its catalytic activity, LPL exerts some inflammatory effects in the arterial wall. It enhances human monocyte adhesion to endothelial cells ${ }^{9}$ and induces TNF- $\alpha$ gene expression and secretion in macrophages. ${ }^{10}$ Also, LPL exerts a synergistic effect on interferon-gamma-induced nitric oxide synthetase expression in macrophages. ${ }^{2,11}$ Further molecular studies are essential to clarify the effect of LPL genetic variants on cytokine release after CPB.

Our study has some obvious limitations. First, the number of the patients is small and increasing the number would increase the statistical power of the study. Second, this study demonstrated only a minor difference in terms of early morbidity and mortality between the groups. Therefore, the results of the present study should be considered as preliminary, and further studies including more high-risk and unstable patients are warranted to provide a more relevant clinical conclusion. Third, it would be beneficial to incorporate other inflammatory cytokines like IL-1, IL-2, and TNF- $\alpha$ and anti-inflammatory cytokine IL-10 into the study. Fourth, CASUS was preferred for the assessment of daily organ dysfunction. More clinical studies are warranted to test its validity and effectiveness in cardiac surgical patients. However, standard ICU scoring systems such as APACHE II and Multiple Organ Dysfunction Score (MODS) were originally designed to estimate the risk for critically ill patients in the ICU, but not specifically for cardiac surgical patients. These scoring systems lack some important postoperative parameters such as lactate and intraaortic balloon pump usage, which were shown to be important determinants of postoperative morbidity and mortality in cardiac surgical patients. Last, some claimed that mechanical ventilation is an important contributing factor to the SIRS in cardiac surgery. ${ }^{22}$ In our study, prolonged ventilation in the S447X noncarrier group might have exaggerated the IL-8 release by attracting additional neutrophils and caused a vicious cycle. Therefore, further studies are warranted.

In conclusion, our results show that LPL S447X genetic variant exerts, at least partially, a resistance to SIRS by decreasing post-CPB levels of IL-8 after CABG. Preoperative LPL S447X genotyping may be beneficial in detecting patients with a genetically determined risk for an exaggerated cytokine response, especially IL-8, and postoperative organ dysfunction after CPB. Alternative treatment strategies such as off-pump CABG and more aggressive ICU management may help to improve the outcome in these at-risk patients. Moreover, insight into the mechanisms underlying the differences in cytokine release among LPL polymorphisms may eventually facilitate the understanding of the pathophysiology of the SIRS after CPB.

\section{References}

1. Hammermeister KE, Burchfiel C, Johnson R, Grover FL. Identification of patients at greatest risk for developing major complications at cardiac surgery. Circulation. 1990;82(5 Suppl):IV380-9.

2. Cremer J, Martin M, Redl H, Bahrami S, Abraham C, Graeter T, et al. Systemic inflammatory response syndrome after cardiac operations. Ann Thorac Surg. 1996;61:1714-20.

3. Paparella D, Yau TM, Young E. Cardiopulmonary bypass induced inflammation: pathophysiology and treatment. An update. Eur J Cardiothrac Surg. 2002;21:232-44

4. Gaudino M, Andreotti F, Zamparelli R, Di Castelnuovo A, Nasso G, Burzotta $\mathrm{F}$, et al. The $-174 \mathrm{G} / \mathrm{C}$ interleukin-6 polymorphism influences postoperative interleukin-6 levels and postoperative atrial fibrillation: is atrial fibrillation an inflammatory complication? Circulation. 2003; 108(Suppl I):II195-9.

5. Drabe N, Zünd G, Grünenfelder J, Sprenger M, Hoerstrup SP, Bestmen L, et al. Genetic predisposition in patients ungergoing cardiopulmonary bypass surgery is associated with an increase of inflammatory cytokines. Eur J Cardiothrac Surg. 2001;20:609-13.

6. Bittar MN, Carey JA, Barnard JB, Pravica V, Deiraniya AK, Yonan N, et al. Tumor necrosis factor alpha influences the inflammatory response after coronary surgery. Ann Thorac Surg. 2006;81:132-7.

7. Grünenfelder J, Umbehr M, Plass A, Bestmann L, Maly FE, Zund G, et al. Genetic polymorphisms of apolipoprotein E4 and tumor necrosis factor beta as predisposing factors for increased inflammatory cytokines after cardiopulmonary bypass. J Thorac Cardiovasc Surg. 2004;128:92-7. 
8. Fisher RM, Humphries SE, Talmud PJ. Common variation in the lipoprotein lipase gene: effects on plasma lipids and risk of atherosclerosis. Atherosclerosis. 1997;135:145-59.

9. Obunike JC, Paka S, Pillarisetti S, Goldberg IJ. Lipoprotein lipase can function as monocyte adhesion protein. Arterioscler Thromb Vasc Biol. 1997;17:1414-20.

10. Kota RS, Ramana CV, Tenorio FA, Enelow RI, Rutledge JC. Differential effects of lipoprotein lipase on tumor necrosis factor-alpha and interferon-gamma-mediated gene expression in human endothelial cells. J Biol Chem. 2005;280:31076-84.

11. Renier G, Lambert A. Lipoprotein lipase synergizes with interferongamma to induce macrophage nitric oxide synthetase mRNA expression and nitric oxide production. Arterioscler Thromb Vasc Biol. 1995;15:392-99.

12. Clee SM, Loubser O, Collins J, Kastelein JJ, Hayden MR. The LPL S447X cSNP is associated with decreased blood pressure and plasma triglycerides, and reduced risk of coronary artery disease. Clin Genet. 2001 60:293-300

13. Gagne SE, Larson MG, Pimstone SN, Schaefer EF, Kastelein JJP, Wilson PWF, et al. A common truncation variant of lipoprotein lipase (S447X) confers protection against coronary heart disease: the Framingham offspring study. Clin Genet. 1999;55:450-4.

14. Hekmat K, Kroener A, Stuetzer H, Schwinger RH, Kampe S, Bennink GB, et al. Daily assessment of organ dysfunction and survival in intensive care unit cardiac surgical patients. Ann Thorac Surg. 2005; 79:1555-62.

15. Humphries SE, Nicaud V, Margalef J, Tiret L, Talmud PJ. Lipoprotein lipase gene variation is associated with a paternal history of premature coronary artery disease and fasting and postprandial plasma triglycerides: the European Atherosclerosis Research Study (EARS). Arterioscler Thromb Vasc Biol. 1998;18:526-34.

16. Day IN, Humphries SE. Electrophoresis for genotyping: microtiter array diagonal gel electrophoresis on horizontal polyacrylamide gels, hydrolink, or agarose. Anal Biochem. 1994;222:389-95.

17. Wittrup HH, Tybjaerg-Hansen A, Nordestgaard BG. Lipoprotein lipase mutations, plasma lipids and lipoproteins, and risk of ischemic heart disease. A meta-analysis. Circulation. 1999;99:2901-7.

18. Kotani N, Hashimoto H, Sessler DI, Muraoka M, Wang JS, O'Connor MF, et al. Neutrophil number and interleukin- 8 and elastase concentrations in bronchoalveolar lavage fluid correlate with decreased arterial oxygenation after cardiopulmonary bypass. Anesth Analg. 2000; 90:1046-51.

19. Rao V, Ivanov J, Weisel RD, Cohen G, Borger MA, Mickle DA. Lactate release during reperfusion predicts low cardiac output syndrome after coronary bypass surgery. Ann Thorac Surg. 2001;71:1925-30.

20. Davies AR, Bellomo R, Raman JS, Gutteridge GA, Buxton BF. High lactate predicts the failure of intraaortic balloon pumping after cardiac surgery. Ann Thorac Surg. 2001;71:1415-20.

21. Damas P, Canivet JL, de Groote D, Vrindts Y, Albert A, Franchimont $\mathrm{P}$, et al. Sepsis and serum cytokine concentrations. Crit Care Med. 1997;25:405-12.

22. Zupancich E, Paparella D, Turani F, Munch C, Rossi A, Massaccesi $\mathrm{S}$, et al. Mechanical ventilation affects inflammatory mediators in patients undergoing cardiopulmonary bypass for cardiac surgery: a randomized clinical trial. J Thorac Cardiovasc Surg. 2005;130: $378-83$.

\section{APPENDIX 1. The cardiac surgery scoring (CASUS) ${ }^{14}$}

\begin{tabular}{|c|c|c|c|c|c|}
\hline Descriptor & 0 points & 1 point & 2 points & 3 points & 4 points \\
\hline $\mathrm{PO}_{2} / \mathrm{FlO}_{2}$ & Extubated & $>250$ & $151-250$ & $75-150$ & $<75$ \\
\hline Serum creatinine $(\mathrm{mg} / \mathrm{dL})$ & $<1.2$ & $1.2-2.2$ & $2.3-4.0$ & $4.1-5.5$ & $>5.5$ \\
\hline Serum bilirubin (mg/dL) & $<1.2$ & $1.2-3.5$ & $3.6-7.0$ & $7.1-14.0$ & $>14.0$ \\
\hline $\mathrm{PAR}=\mathrm{HR} \times \mathrm{CVP} / \mathrm{MAP}$ & $<10.1$ & $10.1-15.0$ & $15.1-20.0$ & $20.1-30.0$ & $>30.0$ \\
\hline Lactic acid (mmol/L) & $<2.1$ & $2.1-4.0$ & $4.1-8.0$ & $8.1-12.0$ & $>12.0$ \\
\hline Platelets $\left(\mathrm{mL} \times 10^{-3}\right)$ & $>120$ & $81-120$ & $51-80$ & $21-50$ & $<21$ \\
\hline Neurologic state & Normal status & Not scored & Confused conversation & Sedated & Diffuse neuropathy \\
\hline Intra-aortic balloon pump & No & Not scored & Not scored & Not scored & Yes \\
\hline Ventricular assist device & No & Not scored & Not scored & Not scored & Yes \\
\hline CVVH/dialysis & No & Not scored & Not scored & Not scored & Yes \\
\hline
\end{tabular}

Increasing abnormality was graded on a scale from 0 to 4 score points: a score of 0 representing normal or minimally deranged function, a score of 4 correlating with a markedly deranged function. $\mathrm{PO}_{2} / \mathrm{FIO}_{2}$, The ratio of arterial $\mathrm{PO}_{2}(\mathrm{~mm} \mathrm{Hg})$ and fractional inspired oxygen concentration; $P A R$, pressure-adjusted heart rate; $H R$, heart rate; $C V P$, central venous pressure; $M A P$, mean arterial pressure; $C V V H$, continuous venovenous hemofiltration. 